\title{
Tractography at 3T MRI of Corpus Callosum Tracts Crossing White Matter Hyperintensities
}

\author{
(DW. Reginold, (D). Itorralba, (D)A.C. Luedke, (D). Fernandez-Ruiz, (D). Reginold, (D). Islam, and (D) A. Garcia
}

\begin{abstract}
BACKGROUND AND PURPOSE: The impact of white matter hyperintensities on the diffusion characteristics of crossing tracts is unclear. This study used quantitative tractography at $3 T$ MR imaging to compare, in the same individuals, the diffusion characteristics of corpus callosum tracts that crossed white matter hyperintensities with the diffusion characteristics of corpus callosum tracts that did not pass through white matter hyperintensities.
\end{abstract}

MATERIALS AND METHODS: Brain T2 fluid-attenuated inversion recovery-weighted and diffusion tensor 3T MR imaging scans were acquired in 24 individuals with white matter hyperintensities. Tractography data were generated by the Fiber Assignment by Continuous Tracking method. White matter hyperintensities and corpus callosum tracts were manually segmented. In the corpus callosum, the fractional anisotropy, radial diffusivity, and mean diffusivity of tracts crossing white matter hyperintensities were compared with the fractional anisotropy, radial diffusivity, and mean diffusivity of tracts that did not cross white matter hyperintensities. The cingulum, long association fibers, corticospinal/bulbar tracts, and thalamic projection fibers were included for comparison.

RESULTS: Within the corpus callosum, tracts that crossed white matter hyperintensities had decreased fractional anisotropy compared with tracts that did not pass through white matter hyperintensities $(P=.002)$. Within the cingulum, tracts that crossed white matter hyperintensities had increased radial diffusivity compared with tracts that did not pass through white matter hyperintensities $(P=.001)$.

CONCLUSIONS: In the corpus callosum and cingulum, tracts had worse diffusion characteristics when they crossed white matter hyperintensities. These results support a role for white matter hyperintensities in the disruption of crossing tracts.

ABBREVIATIONS: $C C=$ corpus callosum; $C C$-WMH tracts $=$ corpus callosum tracts crossing white matter hyperintensities; $F A=$ fractional anisotropy; $M D=$ mean diffusivity; $\mathrm{RD}$ = radial diffusivity; $\mathrm{WMH}=$ white matter hyperintensities; $\mathrm{WMH}$ tracts = tracts crossing white matter hyperintensities

$\mathrm{T}$ he corpus callosum (CC) is the largest commissural tract with $>200$ million axons connecting the cerebral hemispheres. ${ }^{1}$ Atrophy of the CC is a marker of neurodegeneration and has been reported in cerebrovascular disease. ${ }^{2-8}$ White matter hyperintensities (WMH) are high-signal lesions on T2-weighted MR imaging that represent cerebral small vessel disease and have been associated with CC atrophy. ${ }^{7,9-14}$ The reason for changes in the corpus callosum with WMH is unclear. Earlier studies have sug-

Received December 24, 2015; accepted after revision February 16, 2016. From the Departments of Medical Imaging (W.R.) and Life Sciences (J.R.), University of Toronto, Toronto, Ontario, Canada; Memory Clinics (W.R., A.G.), Division of Geriatric Medicine, Department of Medicine, and Centre for Neuroscience Studies (J.I., A.G., A.C.L.), Queen's University, Kingston, Ontario, Canada; Facultad de Medicina, (J.F.-R.), Universidad Nacional Autonoma de Mexico, Coyoacán, Mexico; and Department of Diagnostic Radiology (O.I.), Kingston General Hospital, Queen's University, Kingston, Ontario, Canada.

Please address correspondence to William Reginold, MD, Department of Medical Imaging, University of Toronto, 4th floor, 263 McCaul St, Toronto, ON M5T IW7,

Canada; e-mail: wreginold@qmed.ca

http://dx.doi.org/10.3174/ajnr.A4788 gested that WMH may be an incidental finding and that CC atrophy results from a coexisting disease process. ${ }^{7,10-17}$ For example, WMH are seen with Alzheimer disease, in which CC atrophy can occur by cortical atrophy and subsequent Wallerian degeneration of corpus callosum fibers originating from pyramidal neurons. ${ }^{7,10,13,15,16}$ These studies also suggest that WMH may directly cause CC atrophy by disrupting fibers of the corpus callosum as they are passing through the ischemic lesions in the deep white mater. ${ }^{7,10-17}$

Diffusion tensor imaging can detect early changes in white matter microstructure before atrophy occurs and could clarify the relationship between $\mathrm{WMH}$ and the CC. ${ }^{18}$ In DTI, pathologic processes that alter the structural integrity of tracts lead to changes in water diffusion and mean diffusivity (MD) and radial diffusivity (RD) as well as changes in the directionality of diffusion and fractional anisotropy (FA) ${ }^{18,19}$ In patients with $\mathrm{WMH}$, decreased FA and increased MD were found in the CC. ${ }^{13}$ Another study demonstrated correlations among CC atrophy, the FA/MD of deep white matter, and the FA/MD of the CC. ${ }^{17}$ These results 
Table 1: Demographic and clinical data for participants ${ }^{a}$

\begin{tabular}{lc}
\hline & Total \\
\hline No. & 24 \\
Age (yr) & $70.4 \pm 9.2$ \\
Sex (M/F count) & $8 / 16$ \\
WMH volume (voxels) & $1190 \pm 3030$ \\
MoCA & $27.7 \pm 1.7$ \\
\hline
\end{tabular}

Note:-MoCA indicates Montreal Cognitive Assessment.

a Values are means and SDs unless otherwise noted.

confirm an association between WMH and the entire CC but do not distinguish between the effects of WMH on callosum tracts that cross WMH (CC-WMH) and those that do not cross WMH. If CC-WMH tracts had worse diffusion characteristics than CC tracts not crossing $\mathrm{WMH}$, this feature would support an increased role for $\mathrm{WMH}$ in changes in the corpus callosum.

Tractography is an application of DTI that allows the reconstruction of white matter tracts. ${ }^{18}$ In quantitative tractography, the diffusion characteristics (MD, RD, and FA) along the full trajectory of select fiber tracts can be assessed. ${ }^{18}$ This study used quantitative tractography to compare the diffusion characteristics of CC-WMH tracts with those of CC tracts not crossing WMH. For comparison, this study also performed a similar analysis in tracts that crossed WMH (WMH tracts) compared with those that did not cross WMH (lesion-free tracts) in the cingulum, long association fibers, corticospinal/bulbar tracts, and thalamic projection fibers. We hypothesized that CC-WMH tracts would have worse diffusion characteristics (increased MD and RD and decreased FA) compared with CC tracts not crossing WMH.

\section{MATERIALS AND METHODS \\ Participants}

The study was approved by the research ethics board of Queen's University. All participants provided written informed consent before entering the study. All participants underwent a 3T MR imaging brain scan within 2 weeks of cognitive testing, which included the Montreal Cognitive Assessment, ${ }^{20}$ the Wechsler Memory Scale-III longest span backward, ${ }^{21}$ the Wechsler Memory Scale-III longest span forward, ${ }^{21}$ the Stroop Test, ${ }^{22}$ and Letter-Number Sequencing. ${ }^{21}$ Exclusion criteria included a diagnosis of mild cognitive impairment or dementia or the presence of metallic objects, devices, or conditions unsafe for MR imaging. The inclusion criterion was the presence of white matter hyperintensities on T2 FLAIR imaging. Ninety-one participants had MR imaging scans, of which 24 subjects met the inclusion criteria for the study. The demographic characteristics of the participants are shown in Table 1.

\section{MR Imaging}

All brain imaging was acquired in 1 session on a 3T Magnetom Trio MR imaging system (Siemens, Erlangen, Germany) with a 12-channel head coil. A high-resolution anatomic scan was acquired with a sagittal T1-weighted 3D magnetization-prepared rapid acquisition of gradient echo sequence (FOV, $256 \mathrm{~mm}$; spatial resolution, $1 \times 1 \times 1 \mathrm{~mm}^{3}$; TR, $1760 \mathrm{~ms}$; TE, $2.2 \mathrm{~ms}$; flip angle, $9^{\circ}$; number of sections, 176). An axial T2-weighted 2D fluid-attenuated inversion recovery sequence interleaved scan was acquired for detecting white matter hyperintensities (FOV, 250 $\mathrm{mm}$; voxel size, $1 \times 1 \times 3 \mathrm{~mm}^{3}$; TR, $9000 \mathrm{~ms}$; TE, $79 \mathrm{~ms}$; flip angle, $180^{\circ}$; number of sections, 40). Diffusion tensor imaging data were acquired in 30 directions by using a single-shot echo-planar imaging sequence with 31 volumes of 60 axial sections (b-value $1=$ $0 \mathrm{~s} / \mathrm{mm}^{2}$ and b-value $2=1000 \mathrm{~s} / \mathrm{mm}^{2}$; section thickness, $2 \mathrm{~mm}$; TR/TE, 7800/95 ms; FOV, $256 \times 256 \mathrm{~mm}^{2}$; acquisition matrix, $128 \times 128$, resulting in a resolution of $2 \times 2 \times 2 \mathrm{~mm}^{3}$ ).

\section{Image Analysis}

Analysis was performed with a method described in earlier studies. $^{23,24}$ Briefly, diffusion-weighted images were corrected for eddy current distortions by using the Diffusion Toolbox in the FMRIB Software Library (FSL; www.fmrib.ox.ac.uk/fsl/fslwiki/ $\mathrm{FDT}^{25}$ ). DTI reconstruction of the preprocessed data from FSL was completed with the Diffusion Toolkit 0.5 (TrackVis; www.trackvis.org/dtk). The output included diffusion tensor data, parametric diffusion-weighted imaging, and FA and MD maps. Tracts were created in the Diffusion Toolkit by the Fiber Assignment by Continuous Tracking method. ${ }^{26}$ The subject's T2 FLAIR MR imaging was registered to the diffusion-weighted image map by using 3DSlicer 4.1 (www.slicer.org). Tractography data and registered T2 FLAIR were analyzed with TrackVis. All WMH on axial T2 FLAIR were segmented manually. The borders of individual WMH were identified with windowing of the axial T2 FLAIR sections. Individual WMH were outlined section-bysection on all 40 axial T2 FLAIR sections for each patient by using a mouse-controlled interface on TrackVis. Although there is high intrarater and interrater variability, expert manual segmentation of WMH is the criterion standard for segmenting WMH. ${ }^{27}$ The software generated a single ROI that encompassed all voxels within outlined areas. WMH lesion volume was measured as the total number of voxels that were within the WMH. The locations of WMH were manually classified by using axial, sagittal, and coronal T2 FLAIR reconstructions as periventricular (contiguous with the ventricular system), deep frontal, deep temporal, deep parietal, and deep occipital. ${ }^{28-31}$

For the analysis of the specific white matter tracts, ROIs were manually placed within tracts of interest by using axial and sagittal color FA maps and T2 FLAIR. Tracts of interest were segmented by choosing tracts with any part through the ROIs. Segmented tracts included the corpus callosum, cingulum, long association fibers, corticospinal/bulbar tracts, and thalamic projection fibers (example in Fig 1). For the analysis of WMH tracts, ROIs were combined to select tracts. For example, to segment CC-WMH tracts, tracts with any part through the WMH ROI and any part through the ROI for segmenting the corpus callosum were selected (Fig 1A). The number, mean FA, mean MD, and RD (average of the second and third eigenvalues ${ }^{19}$ ) of WMH tracts were measured. For the analysis of tracts not crossing WMH, ROIs were again combined. For example, to segment tracts within the corpus callosum that did not cross WMH, tracts with no part through the WMH ROI and any part through the ROI for segmenting the corpus callosum were selected (Fig 1A). The number, mean $\mathrm{FA}$, mean $\mathrm{MD}$, and $\mathrm{RD}$ of these lesion-free tracts were measured. The percentage of WMH tracts was calculated as the ratio of the number of WMH tracts to the combined number of WMH tracts and lesion-free tracts. 


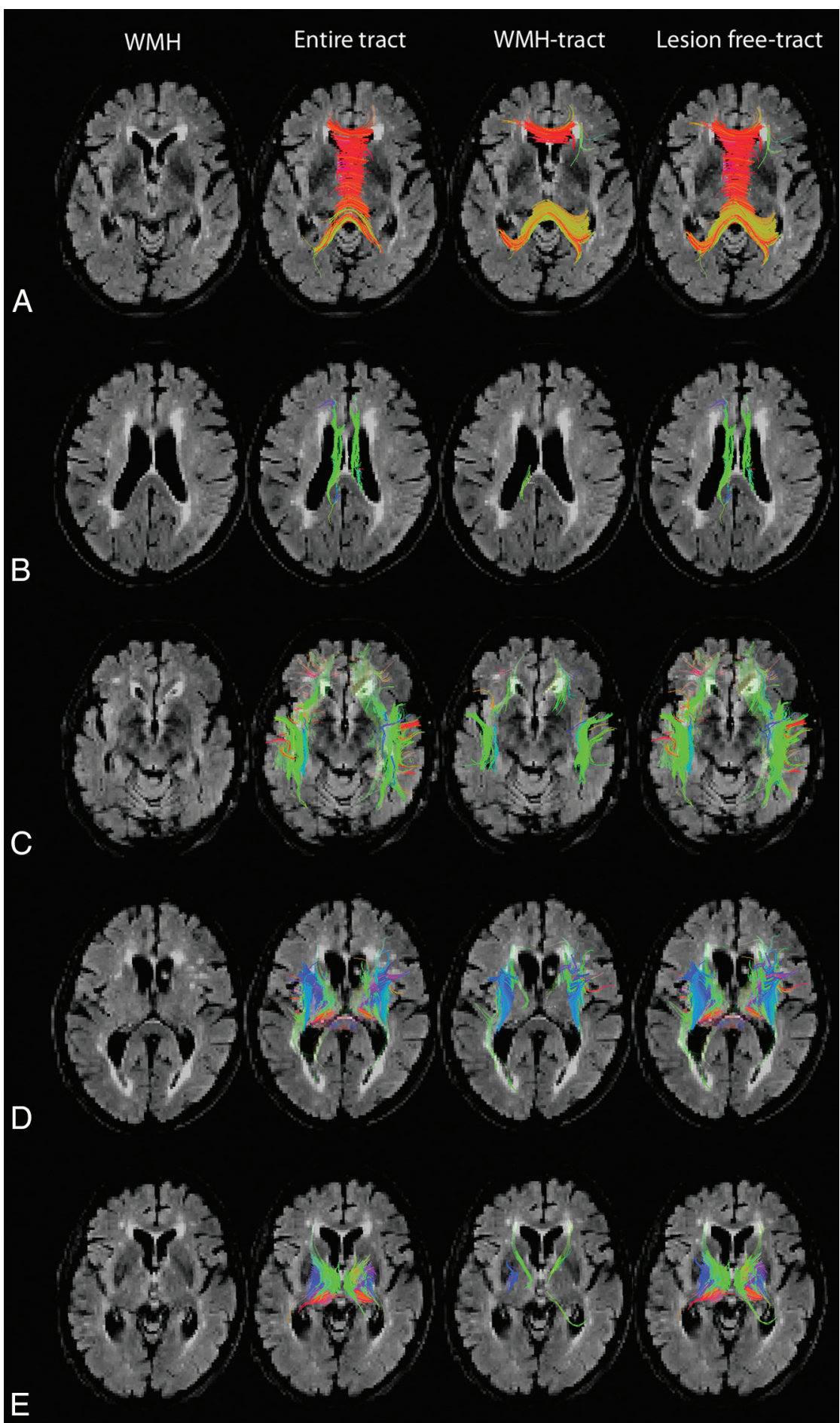

FIG 1. Tract segmentation, WMH tracts, and lesion-free tracts. Axial T2 FLAIR demonstrates white matter hyperintensities, tractography representation of the entire tracts, WMH tracts, and lesion-free tracts in the corpus callosum $(A)$, cingulum $(B)$, association fibers $(C)$, corticospinal/ bulbar tract $(D)$, and thalamic tracts $(E)$.

\section{Statistical Analysis}

StatPlus for Windows (AnalystSoft, Walnut, California) was used for statistical analysis. The nonparametric sign test was used to compare the FA, MD, and RD of tracts crossing WMH with tracts not crossing WMH within subjects. Subjects were included in the analysis when they had tracts crossing WMH. Due to multiple testing, a Bonferroni correction was used to adjust $P$ values. $P$ values $<.01$ were considered statistically significant.

\section{RESULTS}

The demographic characteristics of the participants are described in Table 1 . The age of participants ranged from 56 to 89 years. All participants had periventricular WMH, 18 (75\%) had deep frontal WMH, $11(46 \%)$ had deep parietal $\mathrm{WMH}, 3$ (13\%) had deep occipital $\mathrm{WMH}$, and no participants had deep temporal WMH. WMH were located in the CC in all 24 participants, in the cingulum of $17(71 \%)$ participants, in the association tracts of $21(88 \%)$ participants, in the corticospinal/bulbar tract of $20(83 \%)$ participants, and in the thalamic tracts of $16(67 \%)$ participants. Within these tracts, the mean percentage of fibers crossing $\mathrm{WMH}$ was $14 \% \pm$ $14 \%$ of the CC, $3 \% \pm 5 \%$ of the cingulum, $4 \% \pm 4 \%$ of association fibers, $4 \% \pm 6 \%$ of the corticospinal/bulbar tract, and $1 \% \pm 2 \%$ of thalamic tracts. The CC-WMH tracts and cingulum $\mathrm{WMH}$ tracts crossed only periventricular WMH.

CC-WMH tracts had decreased FA compared with CC lesion-free tracts (Table 2). The percentage decrease in FA of WMH tracts compared with lesionfree tracts was $9 \% \pm 9 \%$ in the CC, $1 \% \pm 13 \%$ in the cingulum, $3 \% \pm 11 \%$ in the association fibers, $6 \% \pm 12 \%$ in the corticospinal/bulbar tracts, and $-2 \% \pm 14 \%$ in the thalamic tracts. There was no significant difference in the MD or RD between CC-WMH tracts and CC lesion-free tracts (Table 2). There was increased RD in cingulum$\mathrm{WMH}$ tracts compared with cingulum lesion-free tracts (Table 2). The percentage increase in the RD of WMH tracts compared with lesion-free tracts was $-2 \% \pm 15 \%$ in the CC, $21 \% \pm 23 \%$ in the cingulum, $1 \% \pm 7 \%$ in the association fibers, $6 \% \pm 15 \%$ in the corticospinal/bulbar tracts, and $5 \% \pm 25 \%$ in the thalamic tracts. For the other major white matter tracts, there were no significant differences in the FA, MD, or RD between tracts crossing $\mathrm{WMH}$ and those not crossing WMH (Table 2).

\section{DISCUSSION}

This is the first tractography study to compare tracts on the basis of whether they crossed WMH. Decreased FA was found in CC$\mathrm{WMH}$ tracts compared with CC tracts not crossing WMH and is 
Table 2: Comparison in major white matter tracts of the fractional anisotropy, mean diffusivity, and radial diffusivity of tracts crossing and not crossing $\mathrm{WMH}^{\mathrm{a}}$

\begin{tabular}{lcccc}
\hline & $\begin{array}{c}\text { No. of Subjects } \\
\text { with Tracts } \\
\text { Crossing WMH }\end{array}$ & Crossing WMH & $\begin{array}{c}\text { Not Crossing } \\
\text { WMH }\end{array}$ & $\begin{array}{c}P \\
\text { Value }\end{array}$ \\
\hline $\begin{array}{l}\text { Fractional anisotropy } \\
\text { Corpus callosum }\end{array}$ & 24 & $0.539 \pm 0.057$ & $0.591 \pm 0.046$ & $.002^{\mathrm{b}}$ \\
$\quad$ Cingulum & 17 & $0.442 \pm 0.076$ & $0.448 \pm 0.047$ & .152 \\
Association fibers & 21 & $0.410 \pm 0.054$ & $0.424 \pm 0.034$ & .093 \\
Corticospinal tract & 20 & $0.446 \pm 0.061$ & $0.477 \pm 0.032$ & .027 \\
$\quad$ Thalamus & 16 & $0.452 \pm 0.073$ & $0.441 \pm 0.032$ & .130 \\
Mean diffusivity & & & & \\
Corpus callosum & 24 & $0.00085 \pm 0.0001$ & $0.000932 \pm 0.0001$ & .053 \\
$\quad$ Cingulum & 17 & $0.0009 \pm 0.0002$ & $0.0008 \pm 0.0001$ & .013 \\
$\quad$ Association fibers & 21 & $0.00079 \pm 0.0001$ & $0.00078 \pm 0.0001$ & .122 \\
Corticospinal tract & 20 & $0.0008 \pm 0.0001$ & $0.0007 \pm 0.0001$ & .047 \\
$\quad$ Thalamus & 16 & $0.00078 \pm 0.0002$ & $0.0008 \pm 0.0001$ & .145 \\
Radial diffusivity & & & & \\
$\quad$ Corpus callosum & 24 & $0.0006 \pm 0.0001$ & $0.0006 \pm 0.0001$ & .140 \\
Cingulum & 17 & $0.0007 \pm 0.0002$ & $0.0006 \pm 0.0001$ & $.001^{\mathrm{b}}$ \\
Association fibers & 21 & $0.0006 \pm 0.0001$ & $0.0006 \pm 0.0001$ & .143 \\
Corticospinal tract & 20 & $0.0006 \pm 0.0001$ & $0.0005 \pm 0.0001$ & .070 \\
Thalamus & 16 & $0.0007 \pm 0.0002$ & $0.0006 \pm 0.0001$ & .126 \\
\hline
\end{tabular}

${ }^{a}$ Values are means and SDs unless otherwise noted.

b Statistically significant.

indicative of greater white matter abnormalities in CC tracts crossing through WMH. WMH were associated with modest white matter abnormalities in the CC ( $8 \%$ reduction in FA in 14\% of all CC tracts). The association between decreased FA in the CC and WMH is consistent with that in earlier studies. ${ }^{13,17}$ Increased $\mathrm{RD}$ was found in the cingulum WMH tracts compared with cingulum lesion-free tracts and is suggestive of greater abnormalities in the myelination of cingulum tracts crossing through WMH. WMH were also associated with modest white matter abnormalities in the cingulum (a $21 \%$ increase in RD in 3\% of all cingulum tracts). Earlier studies have reported involvement of the cingulum by WMH; however, this is the first study to detect increased RD. ${ }^{32-34}$ This tractography study demonstrates that WMH are associated with modest altered diffusion characteristics in crossing tracts.

Earlier studies have suggested that WMH may be incidental markers of a disease process that is disrupting white matter tracts. $^{7,10-17}$ If WMH are markers of a disease process, our results suggest that $\mathrm{WMH}$ are preferentially located on tracts with greater disruption by that disease process. WMH are known to preferentially develop in normal-appearing white matter areas with lower FA than in areas that remain lesion-free. ${ }^{35}$ Earlier studies have also suggested that WMH may disrupt passing tracts. ${ }^{7,10-17}$ Our results support this theory because the structural integrity of the $\mathrm{CC}$ and cingulum tracts was worse when they passed through WMH. WMH consist of mildto-marked demyelination, axonal loss, and astrogliosis and have reduced FA, increased MD, and increased RD within the lesions. ${ }^{35-37}$ The FA of CC-WMH tracts or the RD of cingulum WMH tracts reflects a combination of the diffusion characteristics within the $\mathrm{WMH}$ and the portions of the tracts extending beyond the lesions. If the altered diffusion characteristics within WMH accounted for the differences between $\mathrm{CC}-\mathrm{WMH}$ tracts and CC tracts not crossing WMH or cingulum WMH tracts and cingulum lesion-free tracts, we would have expected to see similar differences in FA and RD between $\mathrm{WMH}$ tracts and lesion-free tracts in the long association fibers, corticospinal/bulbar tracts, and thalamic projection fibers. We were unable to detect any difference between $\mathrm{WMH}$ tracts and lesion-free tracts in any of the other major white matter tracts, suggesting that $\mathrm{WMH}$ may have additional effects on segments of CC-WMH tracts and cingulum WMH tracts outside the lesions. WMH have been previously associated with diffusion abnormalities in normal-appearing white matter closer to WMH. ${ }^{35,36}$ Wallerian degeneration is a mechanism by which a lesion can produce disruption elsewhere along the length of the tract. ${ }^{7,10,13,15,16}$ Decreased FA and increased RD, which we have detected in this study, have been previously associated with Wallerian degeneration. ${ }^{19,38,39}$

Consistent with earlier studies, the periventricular area was the most common location of $\mathrm{WMH}^{28-31}$ In this study, CC-WMH tracts and cingulum WMH tracts crossed only periventricular WMH. In this study, the CC was the tract most likely, in participants, to cross through WMH and had the greatest proportion of fibers crossing through WMH. The greater involvement by WMH and altered FA may explain why atrophy of the CC has been noted earlier with WMH. ${ }^{7,10,11-14}$ In this study, the cingulum, long association fibers, corticospinal/bulbar tract, and thalamic fibers were all shown to cross WMH. The cingulum was the only other tract to demonstrate abnormal diffusion in WMH tracts.

It is unclear why CC-WMH tracts were not associated with increased MD and RD and why cingulum WMH tracts were not associated with decreased FA or increased MD. This lack of information may relate to the small sample size of this study. The altered diffusion characteristics detected were modest in size, and the study may have been underpowered to detect other smaller diffusion changes. The decreased tract involvement by WMH of long association fibers and corticospinal/bulbar tract and thalamic fibers compared with the CC may account for no diffusion abnormalities being detected in these tracts. Another limitation of this study is the accuracy of WMH tract reconstruction. The tractography methodology used in this study relies on FA for fiber tracking and reconstruction. ${ }^{26}$ Some tracts crossing WMH may not have been propagated through the WMH due to the decreased FA within the lesions. This omission may have resulted in some WMH tracts being incorrectly classified as lesion-free tracts. The T2 FLAIR and diffusion tensor images had different resolutions and required registration. Due to errors in alignment, some tracts may have been incorrectly classified as WMH tracts or lesion-free tracts. Another limitation of this study is that diffusion characteristics are an indirect measure of axonal integrity. ${ }^{18}$ While poor diffusion measures are suggestive of structural disruption, they do not necessarily represent functional disconnection.

Despite these limitations, this study suggests the role WMH have in the disruption of CC and cingulum favors tracts crossing through WMH lesions. WMH may be marking injury or causing disruption of crossing tracts. Future studies with larger cohorts should assess longitudinal changes in WMH and the diffusion 
characteristics of the CC and cingulum, as the temporal sequence of changes may improve our understanding of WMH as a cause or marker of tract disruption.

\section{CONCLUSIONS}

Tractography at 3T MR imaging of the corpus callosum demonstrated that tracts that crossed white matter hyperintensities had decreased fractional anisotropy compared with tracts that did not pass through white matter hyperintensities $(P=.002)$. In the cingulum, tracts that crossed white matter hyperintensities had increased radial diffusivity compared with tracts that did not cross white matter hyperintensities $(P=.001)$. These differences in fractional anisotropy and radial diffusivity between tracts crossing and not crossing white matter hyperintensities were not detected in the long association fibers, corticospinal/bulbar tracts, and thalamic projection fibers. Overall, these results support a role for white matter hyperintensities in the disruption of crossing corpus callosum and cingulum tracts.

\section{REFERENCES}

1. Tomasch J. Size, distribution, and number of fibers in the human corpus callosum. Anat Rec 1954;119:119-35 CrossRef Medline

2. Jokinen H, Ryberg C, Kalska H, et al; LADIS group. Corpus callosum atrophy is associated with mental slowing and executive deficits in subjects with age-related white matter hyperintensities: the LADIS Study. J Neurol Neurosurg Psychiatry 2007;78:491-96 Medline

3. Di Paola M, Spalletta G, Caltagirone C. In vivo structural neuroanatomy of corpus callosum in Alzheimer's disease and mild cognitive impairment using different MRI techniques: a review. J Alzheimers Dis 2010;20:67-95 CrossRef Medline

4. Granberg T, Bergendal G, Shams S, et al. MRI-defined corpus callosal atrophy in multiple sclerosis: a comparison of volumetric measurements, corpus callosum area and index. J Neuroimaging 2015;25:996-1001 CrossRef Medline

5. Crawford HE, Hobbs NZ, Keogh R, et al; TRACK-HD Investigators. Corpus callosal atrophy in premanifest and early Huntington's disease. J Huntingtons Dis 2013;2:517-26 CrossRef Medline

6. Wu TC, Wilde EA, Bigler ED, et al. Longitudinal changes in the corpus callosum following pediatric traumatic brain injury. Dev Neurosci 2010;32:361-73 CrossRef Medline

7. Meguro K, Constans JM, Courtheoux P, et al. Atrophy of the corpus callosum correlates with white matter lesions in patients with cerebral ischaemia. Neuroradiology 2000;42:413-19 CrossRef Medline

8. Tomimoto H, Lin JX, Matsuo A, et al. Different mechanisms of corpus callosum atrophy in Alzheimer's disease and vascular dementia. J Neurol 2004;251:398-406 CrossRef Medline

9. Debette S, Markus HS. The clinical importance of white matter hyperintensities on brain magnetic resonance imaging: systematic review and meta-analysis. BMJ 2010;26:341:c3666 CrossRef Medline

10. Vermersch P, Roche J, Hamon M, et al. White matter magnetic resonance imaging hyperintensity in Alzheimer's disease: correlations with corpus callosum atrophy. J Neurol 1996;243:231-34 CrossRef Medline

11. Ryberg C, Rostrup E, Sjöstrand K, et al; LADIS study group. White matter changes contribute to corpus callosum atrophy in the elderly: the LADIS study. AJNR Am J Neuroradiol 2008;29:1498-504 CrossRef Medline

12. Ryberg C, Rostrup E, Paulson OB, et al; LADIS study group. Corpus callosum atrophy as a predictor of age-related cognitive and motor impairment: a 3-year follow-up of the LADIS study cohort. J Neurol Sci 2011;307:100-05 CrossRef Medline

13. Wu XP, Gao YJ, Yang JL, et al. Quantitative measurement to evalu- ate morphological changes of the corpus callosum in patients with subcortical ischemic vascular dementia. Acta Radiol 2015;56: 214-18 CrossRef Medline

14. Yamauchi H, Fukuyama $H$, Shio $H$. Corpus callosum atrophy in patients with leukoaraiosis may indicate global cognitive impairment. Stroke 2000;31:1515-20 CrossRef Medline

15. Teipel SJ, Hampel H, Alexander GE, et al. Dissociation between corpus callosum atrophy and white matter pathology in Alzheimer's disease. Neurology 1998;51:1381-85 CrossRef Medline

16. Teipel SJ, Bayer W, Alexander GE, et al. Progression of corpus callosum atrophy in Alzheimer disease. Arch Neurol 2002;59:243-48 CrossRef Medline

17. Otsuka Y, Yamauchi H, Sawamoto N, et al. Diffuse tract damage in the hemispheric deep white matter may correlate with global cognitive impairment and callosal atrophy in patients with extensive leukoaraiosis. AJNR Am J Neuroradiol 2012;33:726-32 CrossRef Medline

18. Behrens TE, Jbabdi S. MR diffusion tractography. In: Johansen-Berg $\mathrm{H}$, Behrens TE, eds. Diffusion MRI: from Quantitative Measurement to in vivo Neuroanatomy. New York: Elsevier; 2009:333-51

19. Song SK, Sun SW, Ramsbottom MJ, et al. Dysmyelination revealed through MRI as increased radial (but unchanged axial) diffusion of water. Neuroimage 2002;17:1429-36 CrossRef Medline

20. Nasreddine ZS, Phillips NA, Bédirian V, et al. The Montreal Cognitive Assessment, MoCA: a brief screening tool for mild cognitive impairment. J Am Geriatr Soc 2005;53:695-99 CrossRef Medline

21. Wechsler D. Wechsler Memory Scale. 3rd ed. San Antonio: Psychological Corporation; 1987

22. Stroop JR. Studies of interference in serial verbal reactions. Journal of Experimental Psychology 1935;18:643-62 CrossRef

23. Reginold W, Luedke AC, Tam A, et al. Cognitive function and 3Tesla magnetic resonance imaging tractography of white matter hyperintensities in elderly persons. Dement Geriatr Cogn Dis Extra 2015;5:387-94 CrossRef Medline

24. Reginold W, Itorralba J, Tam A, et al. Correlating quantitative tractography at 3T MRI and cognitive tests in healthy older adults. Brain Imaging Behav 2015 Dec 9. [Epub ahead of print] Medline

25. Smith SM, Jenkinson M, Woolrich MW, et al. Advances in functional and structural MR image analysis and implementation as FSL. Neuroimage 2004;23:S208-19 Medline

26. Wiegell MR, Larsson HB, Wedeen VJ. Fiber crossing in human brain depicted with diffusion tensor MR imaging. Radiology 2000;217: 897-903 CrossRef Medline

27. Simões R, Mönninghoff C, Dlugaj M, et al. Automatic segmentation of cerebral white matter hyperintensities using only 3D FLAIR images. Magn Reson Imaging 2013;31:1182-89 CrossRef Medline

28. Fazekas F, Kapeller P, Schmidt R, et al. The relation of cerebral magnetic resonance signal hyperintensities to Alzheimer's disease. J Neurol Sci 1996;142:121-25 CrossRef Medline

29. DeCarli C, Fletcher E, Ramey V, et al. Anatomical mapping of white matter hyperintensities (WMH): exploring the relationships between periventricular WMH, deep WMH, and total WMH burden. Stroke 2005;36:50-55 CrossRef Medline

30. Yoshita M, Fletcher E, Harvey D, et al. Extent and distribution of white matter hyperintensities in normal aging, MCI, and AD. Neurology 2006;67:2192-98 CrossRef Medline

31. Wen W, Sachdev P. The topography of white matter hyperintensities on brain MRI in healthy 60- to 64-year-old individuals. Neuroimage 2004;22:144-54 CrossRef Medline

32. Glodzik L, Kuceyeski A, Rusinek H, et al. Reduced glucose uptake and $\mathbf{A} \boldsymbol{\beta}$ in brain regions with hyperintensities in connected white matter. Neuroimage 2014;100:684-91 CrossRef Medline

33. Tuladhar AM, van Norden AG, de Laat KF, et al. White matter integrity in small vessel disease is related to cognition. Neuroimage Clin 2015;7:518-24 CrossRef Medline

34. Taylor WD, Kudra K, Zhao Z, et al. Cingulum bundle white matter lesions influence antidepressant response in late-life depression: a pilot study. J Affect Disord 2014;162:8-11 CrossRef Medline 
35. de Groot M, Verhaaren BF, de Boer R, et al. Changes in normalappearing white matter precede development of white matter lesions. Stroke 2013;44:1037-42 CrossRef Medline

36. Gouw AA, Seewann A, van der Flier WM, et al. Heterogeneity of small vessel disease: a systematic review of MRI and histopathology correlations. J Neurol Neurosurg Psychiatry 2011;82:126-35 CrossRef Medline

37. Bastin ME, Clayden JD, Pattie A, et al. Diffusion tensor and magnetization transfer MRI measurements of periventricular white mat- ter hyperintensities in old age. Neurobiol Aging 2009;30:125-36 CrossRef Medline

38. Sun SW, Liang HF, Cross AH, et al. Evolving Wallerian degeneration after transient retinal ischemia in mice characterized by diffusion tensor imaging. Neuroimage 2008;40:1-10 CrossRef Medline

39. Thomalla G, Glauche V, Weiller C, et al. Time course of Wallerian degeneration after ischaemic stroke revealed by diffusion tensor imaging. J Neurol Neurosurg Psychiatry 2005;76:266-68 CrossRef Medline 